Association for Information Systems AIS Electronic Library (AISeL)

\title{
Application of Artificial Bee Colony Algorithm in Vehicle Routing Problem With Time Windows
}

Cong Chen

Wuhan Polytechnic University, 2325995450@qq.com

Kang Zhou

Wuhan Polytechnic University, zhoukang65@whpu.edu.cn

Follow this and additional works at: http://aisel.aisnet.org/confirm2018

\section{Recommended Citation}

Chen, Cong and Zhou, Kang, "Application of Artificial Bee Colony Algorithm in Vehicle Routing Problem With Time Windows" (2018). CONF-IRM 2018 Proceedings. 14.

http://aisel.aisnet.org/confirm2018/14 


\title{
APPlication OF ARTIFICIAL BEE COLONY AlgorithM IN Vehicle Routing Problem With Time Windows
}

\author{
Cong Chen \\ Wuhan Polytechnic University \\ 2325995450@qq.com
}

\author{
Kang Zhou* \\ Wuhan Polytechnic University \\ zhoukang65@whpu.edu.cn
}

\begin{abstract}
:
In order to improve the accuracy of the artificial bee colony algorithm (ABC) on vehicle routing problem with time window (VRPTW), This paper makes the following improvements to the $\mathrm{ABC}$ :We introduce three kinds of neighborhood search methods, In the leader bee and follower bee search stage,we changing the single search mode into a three-way search method,which improves the optimization depth of the algorithm.Conducting multiple neighborhood searches of new food sources generated by the scouter bee and proceeding to the next iteration has enhanced the survival of new food sources and increased the diversity of populations. The global optimal solution is recorded by setting and updating the bulletin board. Simulation experiments show that the improved discrete $\mathrm{ABC}$ algorithm has obvious advantages in solving large-scale VRPTW. Therefore, the improved discrete ABC algorithm has great potential and application value in solving VRPTW.
\end{abstract}

\section{Key words:}

VRPTW; discrete artificial bee colony algorithm; neighborhood search

\section{Introduction}

The Vehicle Routing Problem with Time Windows (VRPTW) is a well-known combinatorial optimization problem(Savelsbergh,1985).Compared to VRP(Szeto,2011), VRPTW increases the time window, So VRPTW is closely related to people's real life(Zhu,2001).How to solve VRPTW effectively has always been one of the hot and difficulties spots in operations research. VRPTW is mainly divided into precise algorithm(Da,2017) and heuristic algorithm(Liu,2005).With the continuous development of artificial intelligence field(Nourossana,2012), people have a new direction for solving VRPTW. Currently, The heuristic algorithm is usually based on a specific algorithm, starting from an initial solution, in the case of satisfying the algorithm constraints and VRPTW constraints on the initial solution of the initial iterative optimization, and ultimately search for the desired solution to the problem. The heuristic algorithm has a good advantage in solving the VRPTW problem because the heuristic algorithm has a short running time, high precision and fast convergence. The sub heuristic optimization algorithm with better VRPTW effect is mainly Genetic Algorithm(Ma,2014),,Partical Swarm Optimization(Zhang,2008), Ant Colony Optimization(Rizzoli,2017), Artificial Fish-Swarm Algorithm(Liu,2010), Ariificial Neural Networks(Wang,2015), Tabu Search(Gupta,2002),Simulated Annealing(Yang,2006), and a hybrid algorithm constructed by fusing several algorithms to overcome some of the inherent shortcomings of the algorithm(Zhang,2017). And artificial bee colony algorithm(Karaboga,2005) is a mimic bee breeding, honey and other behavior(Yang,2009) of the emerging group of intelligent optimization algorithm. The algorithm is simple and easy to 
implement, has few parameters, fast convergence speed, and easy to integrate with other algorithms to form a new hybrid algorithm, but the shortcoming is easy to fall into the local optimal. In general, ABC algorithm has a good potential in solving VRPTW.

\section{VRPTW and Standard ABC Algorithm}

\subsection{Mathematical Model of VRPTW}

VRPTW is a combinatorial optimization problem that increases the time window constraints based on the basic VRP(Yang,2010), the problem can be described as: seeking a number of vehicles from the distribution center, to meet the customer point of time window constraints, their own load constraints, customer service unique constraints under the conditions of service all customer points, and finally return to the distribution center in the minimum number of vehicles under the premise of the path to achieve the shortest vehicle routing arrangements, the mathematical model of VRPTW are as follows(Wang,2014).

$V=\{1,2, \cdots, n\}$ represents the number of customer points; $C=\left\{c_{i j} \mid(i, j) \in A\right\}$ represents the vehicle distance between the customer point $i$ and $j ; K=\{1,2, \ldots, m\}$ represents vehicle number; $g_{i}$ represents the amount of customer point i requirements; $q$ represents the carrying capacity of each vehicle; $\left[a_{i}, b_{i}\right]$ represents the time window for the first $i$ client.

$$
\begin{aligned}
& y_{k i}= \begin{cases}1 & \text { Customerrequirement s for pointI are completedby vehicleK } \\
0 & \text { Other }\end{cases} \\
& x_{i j k}= \begin{cases}1 & \text { ThevehicleK travelfrompointI topoint J } \\
0 & \text { Other }\end{cases} \\
& \min F(x)=p_{1} M+p_{2} \sum_{k} \sum_{i} \sum_{j} c_{i j} x_{i j k} \quad(k \in m, i \in n, j \in n) \\
& \text { s.t. } \begin{cases}\sum_{i} g_{i} y_{k i} \leq q & \forall k \\
\sum_{k}^{k} y_{k i}=1 & i \in V \\
\sum_{j} x_{i j k}=y_{k i} x_{i j k}=y_{k j} & i \in V ; \forall k \\
\sum_{i} \sum_{i, j \in S^{*} x_{i j k}=|S|-1} & S \subseteq V ; \quad \forall k \\
a_{i}+t_{i j} \leq b_{i} & i, j \in V \\
x_{i j k} \in(0,1) & i, j \in V \\
y_{k i} \in(0,1) & i, j \in V\end{cases}
\end{aligned}
$$

p1 and p2 are integers and p1 > p2, so that the minimum number of vehicles is guaranteed to be the first optimization target, and the shortest path value is second, (2-2) is the minimum number of vehicles, $|\mathrm{S}|$ is the number of customer points in the set $S$, and (2-2) is the vehicle load limit. The formula (2-3) shows the customer service uniqueness limit; (2-4) (2-6) is to ensure the formation of Hamiltonian circuit; type (2-7) for the time window constraints.

\subsection{Standard Artificial Bee Colony Algorithm}

The artificial bee colony algorithm (ABC) is an emerging group intelligent optimization 
technology that was proposed by Karaboga scholars of Ergiyes University in Turkey in 2005, In the ABC algorithm, the food source is mapped into the solution of the target problem model, after the continuous division of labor, the cycle search is carried out, and finally the global optimal solution is found. The $\mathrm{ABC}$ algorithm divides the swarm into three types: the leader bee, the follower bee, and the scouter bee. The leader bee passes the food source information to be sent to the follower bee through a special signal. The follower bee selects the food source by a certain probability based on the information and searches in the corresponding neighborhood. If the food source is not updated within the specified number of times, the source will be discarded and the corresponding leader bee will become a new crop for scouter bee.

Assuming that the initial solution of the ith bee is $x i=(x i 1, x i 2, \cdots \cdots, x i D), i(i \in 1,2, \ldots, S N), \mathrm{SN}$ is the size of the colony, $j(j \in 1,2, \ldots, D)$. D is the dimension of the problem.

The leader bee and follower bee produce a new solution to the neighborhood of the food source according to the formula (2-10).

$$
v_{i j}=x_{i j}+\operatorname{ramd}(-1,1)_{i j}\left(x_{i j}-x_{k j}\right)
$$

The follower bee perform a neighborhood search of food sources according to formula (2-11)

$$
p_{i}=\frac{f_{i}}{\sum_{i}^{S N} f i}
$$

The scouter bees use the formula (2-12) to produce new food source instead of discarded food source.

$$
x_{i j}=x_{\min }+\operatorname{rand}(-1,1)_{i j}\left(x_{i j}-x_{k j}\right)
$$

\section{Improved discrete artificial bee colony algorithm for VRPTW}

\subsection{Increased neighborhood search}

In the standard discrete ABC algorithm, the leader bee and the follower bee are only used in a variety of neighborhood search methods, which will lead to the colony of the food source search depth is not enough to find a satisfactory solution.In the neighborhood transformation of the selected food sources, the leader bee and follower bee use one of the three neighborhood search methods shown in Fig 3.1-3.3 to generate a new solution. Compare the number of vehicles and fitness values of new solutions and old solutions, The solution to the small number of vehicles left,If the number of vehicles is the same, then the solution with small fitness will be left.

The specific mode of operation is: assume that the rout of current colony is $X_{i}=\left(\begin{array}{llll}1 & 4 & 3 & 5\end{array} 2\right)$.

1)Take two positions $j_{1}, j_{2}\left(j_{1}, j_{2} \in\{1,2, \ldots, D\}, j_{1} \neq j_{2}\right)$, the value of the $j_{1}$-th position is exchanged with the value of the $j_{2}$-th position in the food source $X_{i}$, so that a new food source $X_{i}{ }^{\prime}=\left(\begin{array}{lll}1 & 5 & 3\end{array}\right.$ 42 ) can be obtained after the transformation. 


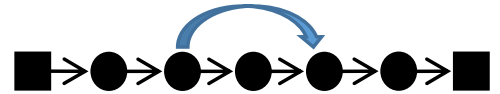

$\begin{array}{lllllll}0 & 1 & 4 & 3 & 5 & 2 & 0\end{array}$

(a)Pre exchange path
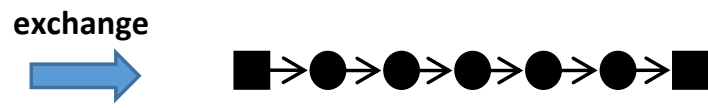

$\begin{array}{lllllll}0 & 1 & 5 & 3 & 4 & 2 & 0\end{array}$

(b)Post exchange path

Fig 3.1 Exchange two elements

2)Take two positions $j_{1}, j_{2}\left(j_{1}, j_{2} \in\{1,2, \ldots, D\}\right.$ 且 $\left.j_{1} \neq j_{2}\right)$, in the food source $X_{i}$, the value of the $j_{1}$-th position is inserted in front of the value of the $j_{2}$-th position, so that a new food source $X_{i}{ }^{\prime}$ $=\left(\begin{array}{lllll}1 & 3 & 5 & 4 & 2\end{array}\right)$ can be obtained after the transformation.

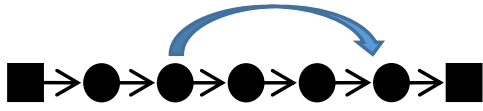

$\begin{array}{lllllll}0 & 1 & 4 & 3 & 5 & 2 & 0\end{array}$

(a)pre insert path

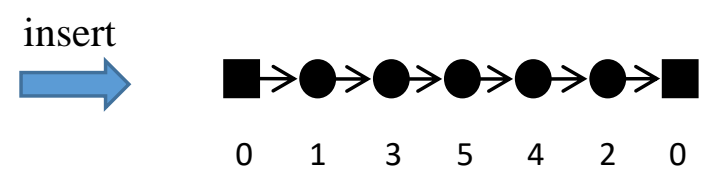

(b)post insert path

Fig 3.2 An element is inserted before another element

3)Take two positions $j_{1}, j_{2}\left(j_{1}, j_{2} \in\{1,2, \ldots, D\}\right.$ 且 $\left.j_{1} \neq j_{2}\right)$, in the food source $X_{i}$, all values between the position $j_{1}$ and the $j_{2}$ position are exchanged at the beginning and the end, so that a new food source $X_{i}{ }^{\prime}=\left(\begin{array}{lllll}5 & 3 & 4 & 1 & 2\end{array}\right)$ can be obtained after the transformation.

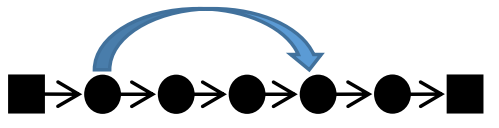

$\begin{array}{lllllll}0 & 1 & 4 & 3 & 5 & 2 & 0\end{array}$

(a)path before reversion
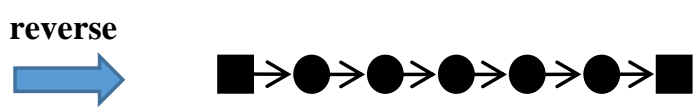

$\begin{array}{lllllll}0 & 5 & 3 & 4 & 1 & 2 & 0\end{array}$

(b)path after reversion

Fig 3.3 reversal between two elements

\subsection{The setting of bulletin board}

In the standard discrete $\mathrm{ABC}$ algorithm, with the increase in the number of iterations, the best food source may no longer be transformed and abandoned by scouter bees, resulting in this better solution is not recorded, in order to avoid the optimal solution is not abandoned, in the program, we use a function to set the bulletin board. After limit times iteration, Compare the current number of vehicles and fitness values for the best solution and bulletin board solution, The solution to the small number of vehicles left,If the number of vehicles is the same, then the solution with small fitness will be left, which can make the bulletin board records are always the best global solution.

\subsection{Treatment of food sources by scouter bee}

In the program, we set an array to record the changes of each food source for any limit iteration, set a limit value, and discard the food source that does not reach the limit value after the iteration and produce a new food. In the standard discrete ABC algorithm, the new food source is generated and enters the next limit iteration along with other sources of food that have not been discarded, but these new sources of food are randomly generated, so the fitness value will be compared poor, it takes a lot of iterations to narrow the gap with the source of food that has not been abandoned, so these new food source in the roulette in the 
competitiveness will be relatively low, easy to fall into the local optimal. In the improved algorithm proposed in this paper, after the new food source is generated, the detection bee will arbitrarily select an iterative limit times for the new food source according to the neighborhood search method of the graph 3.1-3.3. In this way, the new food source fitness will be greatly improved, and the competitiveness will be greatly enhanced in the later iterations.

\section{Simulation experiment and data analysis}

The program uses C language in Visual Studio 2013, using Windows7 64 bit operating system, processor is the third generation of Intel core i3-3110M dual-core, running memory 6GB. We tested many VRPTW examples in the standard database(http://web.cba.neu.edu/ msolomon/problems.htm), divided into six data sets R1, R2, $\mathrm{C} 1, \mathrm{C} 2, \mathrm{RC} 1$ and $\mathrm{RC} 2$. The clients in the set R1 and R2 are evenly distributed, and the clients in the set $\mathrm{C} 1$ and $\mathrm{C} 2$ are clustered and the clients are semi-aggregated in RC1 and RC2. In addition, the set $\mathrm{R} 1, \mathrm{C} 1, \mathrm{RC} 1$ has a small time window and a small vehicle capacity, and the sets R2, C2 and RC2 have a large time window and a large vehicle capacity.

\subsection{Comparison of standard discrete artificial bee colony algorithm and improved discrete artificial bee colony algorithm}

In addition, we compare and analyze the standard discrete $\mathrm{ABC}$ algorithm and the improved discrete ABC algorithm according to different examples, see Table 4.1.

\begin{tabular}{ccccccc}
\hline \multirow{2}{*}{ Examples } & \multicolumn{3}{c}{ Standard Artificial Bee Colony Algorithm } & \multicolumn{3}{c}{ Improved Artificial Bee Colony Algorithm } \\
\cline { 2 - 6 } & vehicles & optimal value & average & vehicles & optimal value & average \\
\hline C102.100 & 12 & 1210.6 & 1305.0 & $\mathbf{1 0}$ & $\mathbf{8 2 8 . 9}$ & 876.3 \\
C105.100 & 12 & 1016.9 & 1115.2 & $\mathbf{1 0}$ & $\mathbf{8 2 8 . 9}$ & 857.4 \\
C108.100 & 12 & 1045.3 & 1205.1 & $\mathbf{1 0}$ & $\mathbf{8 2 8 . 9}$ & 879.2 \\
C201.100 & 9 & 1251.8 & 1386.0 & 4 & 618.5 & 669.9 \\
C202.100 & 8 & 1016.2 & 1112.7 & 4 & 626.8 & 666.0 \\
R101.100 & 27 & 1904.6 & 1956.0 & $\mathbf{2 0}$ & $\mathbf{1 6 6 6 . 8}$ & 1683.7 \\
R102.100 & 22 & 1690.9 & 1743.0 & 19 & $\mathbf{1 4 9 6 . 3}$ & 1513.7 \\
R201.100 & 12 & 1426.2 & 1512.3 & 9 & 1223.6 & 1247.6 \\
RC101.100 & 22 & 1990.2 & 2055.1 & 17 & $\mathbf{1 6 9 7 . 8}$ & 1734.0 \\
RC201.100 & 11 & 1638.0 & 1744.8 & 8 & 1337.8 & 1408.7 \\
\hline
\end{tabular}

Table 4.1 Comparison of Standard and Improved Discret ABC Algorithm for VRPTW Results

Results Analysis: through the experimental results of different examples, we can easily find that the improved discrete $\mathrm{ABC}$ algorithm is better than the standard discrete $\mathrm{ABC}$ algorithm in solving the VRPTW problem. The precision of the standard discrete ABC algorithm is very poor. Every aspect are far inferior to the improved discrete $A B C$ algorithm. which indicates that the standard discrete $\mathrm{ABC}$ algorithm is easy to fall into the local optimum, a new food source has no advantage in the competition. In the improved discrete ABC algorithm, the new food source in the scouter bee stage for multiple neighborhood search, increasing the competitiveness of new food sources, easier to jump out of the local optimal, increasing the breadth of algorithm search. Through the comparison we can draw the following conclusions: 
the improved discrete $\mathrm{ABC}$ algorithm has excellent performance in convergence rate and solving precision, which solves the problem that the standard discrete $\mathrm{ABC}$ algorithm is easy to fall into the local optimum, the stability is poor, the search ability is not strong, which shows that our improvement direction is correct.

\subsection{Comparison of Improved Artificial Bee Colony Algorithm and Database Optimal Solution}

The main parameters of the paper are taken $S N=50$, limit $=10000, M C N=500000, D=100$, we use several different kinds of VRPTW examples to test the improved discrete $A B C$ algorithm. The specific data test results in Table 4.3

\begin{tabular}{ccccccc}
\hline \multirow{2}{*}{ Example } & \multicolumn{3}{c}{ Improved discrete artificial bee algorithm } & \multicolumn{3}{c}{ database optimal solution } \\
\cline { 2 - 7 } & vehicles & routevalue & deviation & average & vehicles & bestvalue \\
\hline C101.100 & $\mathbf{1 0}$ & $\mathbf{8 2 8 . 9}$ & 0.001 & 841.2 & 10 & 827.3 \\
C105.100 & $\mathbf{1 0}$ & $\mathbf{8 2 8 . 9}$ & 0.001 & 857.4 & 10 & 827.3 \\
C108.100 & $\mathbf{1 0}$ & $\mathbf{8 2 8 . 9}$ & 0.001 & 879.2 & 10 & 827.3 \\
C109.100 & $\mathbf{1 0}$ & $\mathbf{8 2 8 . 9}$ & 0.001 & 898.9 & 10 & 827.3 \\
C201.100 & 4 & 618.5 & 0.049 & 669.9 & 3 & 589.1 \\
R101.100 & $\mathbf{2 0}$ & $\mathbf{1 6 6 6 . 8}$ & 0.017 & 1683.7 & 20 & 1637.7 \\
R102.100 & 19 & 1496.3 & 0.020 & 1513.7 & 18 & 1466.6 \\
R201.100 & 9 & 1213.6 & 0.061 & 1248.5 & 8 & 1143.2 \\
RC102.100 & 15 & 1512.5 & 0.037 & 1557.5 & 14 & 1457.4 \\
RC201.100 & $\mathbf{8}$ & 1337.8 & 0.060 & 1408.7 & 9 & 1261.8 \\
\hline
\end{tabular}

Table 4.3 Improved discrete artificial bee algorithm for solving VRPTW experimental results

Results Analysis: from Table 4.3 we can see that for the set C1, our algorithm can achieve the same optimal value in the database, for the collection $\mathrm{RC} 1$, the error rate is basically less than $5 \%$.For the set R1, the improved discrete artificial bee colony algorithm can be counted within $2 \%$. For the set RC2, the number of vehicles is less than the optimal number of vehicles in the database. These example illustrate that the improved discrete $\mathrm{ABC}$ algorithm is still a very competitive algorithm for solving VRPTW. It also has excellent performance in terms of global optimization, convergence speed and solving accuracy and shows that the algorithm has good stability. However, for R2 and C2 examples, our algorithm is not satisfactory, Our algorithm in solving large time windows and large vehicle capacity of the set of examples is not very good, easy to fall into the local optimal, there is a certain gap between the path value and the database in the optimal path value. This also requires our further improvement.

\section{Concluding remarks}

In order to avoid the standard artificial bee colony algorithm local search ability is not strong,the search speed is too slow and it is easy to fall into the local optimum. We proposed an improved discrete artificial bee algorithm to solve VRPTW.Through the experiment of different scale examples, the efficiency of the proposed algorithm is verified. In the later study, how to choose different coding methods and data structures, how to deal with the specific constraints of the problem, update the neighborhood search mode, enhance the local search 
capabilities to reduce computing time and improve the speed of the algorithm, which will be my effort direction.

\section{Acknowledgment}

The work was supported by the Special Scientific Research Fund of Food Public Welfare Profession of China (201513004-3), subproject of the National Key Research and Development Program of China (2017YFD0401102-02), the Guiding Scientific Research Project of Hubei Provincial Education Department (B2017078) and the Humanities and Social Sciences Fund Project of Hubei Provincial Education Department (17Y071). Kang Zhou is corresponding author.

\section{References}

Da, J. Zheng, L.(2017). Study on Exact Algorithm for Vehicle Routing Problem with Time Window[J].Logistics Technology, 36 (6) :95-99.

David Mestera,Olli Braysy(2005).Active guided evolution strategies for large-scale vehicle routing problems with time windows[J]. Computers \& Operations Research, 32(6), 1593-1614.

Gupta D. K(2002). Tabu Search for Vehicle Routing Problems (VRP) [J]. International Journal of Computer Mathematics, 79(6), 693-701

Karaboga D(2005). An idea based on honey bee swarm for numerical optimization[R]. Kayseri, Turkey: Erciyes University

Liu Y(2010). Improved Artificial Fish Swarm Algorithm for Vehicle Routing Problem with Backhaul and Fuzzy Demand[J]. PR\&AI, 23(4), 560-564

Liu, Y. Xuan, H.(2005). Summarizing Research on Models and Algorithms for Vehicle Routing Problem[J]. Journal of Industrial Engineering/Engineering Management, 19(1).

Ma, X. Li, H.(2014). A New Genetic Algorithm for the Capacity Constraints Vehicle Routing Problem[J]. Advances in Applied Mathematics. 03 :222-230.

Nourossana, S. Erfani, H.,et al(2012). BEE COLONY SYSTEM:PRECISENESS AND SPEED IN DISCRETE OPTIMIZATION[J].International Journal on Artificial Intelligence Tools, 21(1), 1250006-1250016.

Rizzoli A. E, Montemanni R, Lucibello E, et al(2007). Ant colony optimization for real-world vehicle routing problems[J], Swarm Intell, 1(2), 135-151.

Savelsbergh, M. W. P.(1985).Local Search in Routing Problems with Time Windows[J]. Armuals of Operations Research, 4, 285-305.

Szeto, WY; Wu, Y; Ho, SC (2011). An artificial bee colony algorithm for the capacitated vehicle routing problem.European Journal of Operational Research 215, no.1: 126-135.

Wang, D. Zheng, P. (2015).Chaotic neural network-based solution to vehicle routing problem[J]. Computer Integrated Manufacturing Systems. 11(12)

Wang, Z. Xia, H.(2014).An artificial bee colony algorithm for the vehicle routing problem[J].Computer Engineering and Science.36(6)

Yang, J. Ma, L.(2009).Wasp colony algorithm for vehicle routing problem with time windows[J].Application Research of Computers. 46(5), 4009-4010

Yang, J. Ma, L. (2010). Wasp colony algorithm for vehicle routing problem[J]. Computer Engineering and Applications, 46(5), 214-216

Yang, Y. Lang, M. Hu, S(2006), Study on Model and Improved Simulated Annealing 
Alogrithm for Vehicle Routing Problem with Time Windows[J]. Journal of Industrial Engineering Management, 20(3), 104-107

Yao, B Z, Hu P,Zhang M H, and Wang S (2013).Artificial bee colony algorithm with scanning strategy for the periodic vehicle routing problem. Simulation 89, no. 6: 762-770.

Zhu, C. LIU, M. WU ,C.(2001).Review of Vehicle Routing Problem in Supply China[J]. CIMS, 7(11), 1-6.

Zhang, D. Cai, S. Ye, F. et al.(2017)A hybrid algorithm for a vehicle routing problem with realistic constraints[J]. Information Sciences. s 394-395(C) :167-182

Zhang, L. Lei, X.(2008). Comparison and Analysis of Several Improved PSO Algorithms in Solving the Vehicle Routing Problem with Time Window[J]. Computer Engineering and Science .30(12) 